\title{
Risk and Variability in Geotechnical Engineering
}

\author{
Editorial
}

The inherent variability of soils and other geo-materials leads to uncertainties: in particular, with regard to on-site material conditions, the behaviour of materials (and movement of fluids) at the macro-scale, and overall response, including soil-structure interaction. There is therefore an associated risk with any geotechnical design.

Geotechnical design has usually been based on a deterministic approach. This involves the identification of material zones; the use of representative material properties for each zone, such as a mean or lower bound strength; and a single analysis leading, for instance, to a global factor of safety. However, this approach takes no account of the variability within zones and the probability of failure is not quantified.

An alternative and more recent approach is to express geo-structural performance within a probabilistic or stochastic framework. In contrast to deterministic analysis, this may involve the use of all data from a site, with the material properties for each zone being defined as probability density functions (rather than single values). These data may then be used in assessments of structural performance, which may involve multiple analyses (as in a Monte Carlo simulation). This leads to probabilistic definitions of response. For example, it may lead to the determination of reliability (the probability that failure will not occur), from which reliability-based factors of safety and characteristic values may be derived.

The simplest probabilistic methods only consider pointwise variability, defined by the mean and coefficient of variation of the property value, while others use variance reduction techniques to account for spatial averaging. More advanced stochastic methods use a spatial correlation parameter, often referred to as the scale of fluctuation, for modelling the detailed spatial and anisotropic nature of soil variability.

The last 30 years have seen pioneering work in the application of probabilistic and stochastic methods in geotechnical engineering, and there are many researchers worldwide who have made significant contributions in this field. Despite this, it has remained a minority discipline, due partly to the perceived computational and material data requirements, and partly due to an understandable reluctance (on the part of engineers) to move away from long-established and successful methods of design. However, rapid developments in computer technology have increased the accessibility of this type of analysis, as has the development of computationally less intensive formulations. Meanwhile, new challenges in geotechnical engineering are coming to the fore, raising the possibility of a more prominent role for stochastic-based methods in the future.

In particular, at the start of the 21 st century, engineers are faced with increasingly complicated ground conditions, including the effects of land contamination. There is a demand for an increased understanding of the processes involved in geotechnical (and geoenvironmental) problems, for associated risks to be quantified explicitly and for design codes to address these needs. Such problems need to be investigated probabilistically/stochastically. Indeed, this is recognised in recent design codes, including Eurocode 7 which states that 'characteristic values of soil and rock properties shall take account of the variabilities of the property values' (CEN, 1994). Controversially, although statistical methods are suggested as a possible way forward, there exists little guidance as to how this should be achieved.

The premise here is that probabilistic and stochastic methods lead to more realistic definitions of response, reflecting the variable nature of the materials being analysed. They also lead to an improved understanding of how soils behave and, ultimately, to economy of design. However, they also involve the use of new technologies and ideas that are unfamiliar to many geotechnical engineers. It is against this background that the Géotechnique Editorial Advisory Panel has organised a Symposium in Print on 'Risk and Variability in Geotechnical Engineering', with a view to promoting further research and increased awareness in this important subject area.

The Symposium in Print focuses on the fact that soils and rocks are variable materials, and that taking account of this geologic variability appears crucial for a proper understanding of certain geotechnical problems. The aims of the Symposium are twofold: firstly, to consider the nature, measurement and statistical characterisation of soil variability; secondly, to demonstrate how probabilistic and stochastic methods of analysis may be used to assess the effects of soil variability and, via increased understanding, influence geotechnical design and construction.

The call for papers was issued in September 2003 and attracted great international interest, with a total of 63 papers being offered. Of these, 17 have been selected for publication in the February and March 2005 issues of Géotechnique, including contributions from Australia, Canada, Europe, Hong Kong, Singapore and the USA. The papers vary from the more 
theoretical to those of a practical nature, including case histories. Applications include foundations, retaining structures, slopes and soil-structure interaction. These papers, along with the one-day Symposium to be held in May, are an ideal opportunity for readers to be briefed on the latest developments in a new and rapidly developing area of geotechnical engineering.

I wish to thank the members of the Symposium in Print Organising Committee for their hard work. Their detailed feedback for authors deserves special mention. We are also grateful to the many reviewers of the Symposium papers.
REFERENCE

CEN (European Committee for Standardisation) (1994). Eurocode 7: Geotechnical design. Part 1: General rules, ENV 1997-1. Brussels: CEN.

\section{A. Hicks}

Symposium in Print Sub-Committee Chairman

Dr Michael Hicks, The University of Manchester

Géotechnique Advisory Panel Member

Dr Lidija Zdravkovic, Imperial College London

External Members

Professor Denys Breysse, University of Bordeaux

Dr Trevor Orr, Trinity College Dublin

Professor Ian Pyrah, Napier University 